\title{
Cuestionarios para la detección de trastornos respiratorios del sueño
}

\author{
Questionnaires for the detection of sleep-related \\ breathing disorders
}

Eduardo Borsini', 2, Magalí Blanco', ,',Alejandro Salvado', Glenda Ernst', ${ }^{3}$

\section{RESUMEN}

El síndrome de apneas de sueño (SAHOS) presenta una elevada prevalencia. El diagnóstico puede realizarse mediante la realización de estudios de polisomnografía o poligrafía respiratoria. Sin embargo, algunos cuestionarios pueden ser utilizados para identificar a los pacientes con alta probabilidad clínica de presentar trastornos respiratorios del sueño.

El objetivo de este trabajo es describir la utilidad de los cuestionarios de Berlín;Epworth y el cuestionario de STOP-BANG (SBQ) para determinar riesgo de padecer SAHOS. Se menciona además un cuestionario validado denominado GAIAS. Este último ha sido desarrollado por un equipo argentino e involucra un cuestionario detallado de síntomas y factores que propician el desarrollo de esta enfermedad.

La utilidad de estos cuestionarios radica en la interpretación de cada uno de ellos que se detalla en este trabajo.

Palabras clave: somnolencia, cuestionarios, apneas de sueño.

\section{ABSTRACT}

The obstructive sleep apnea syndrome (OSAHS) has a high prevalence. The diagnosis can be made by carrying out studies of polysomnography or respiratory polygraphy. However, some questionnaires can be used to identify patients with a high clinical probability of presenting respiratory sleep disorders.

The aim of this paper is to describe the usefulness of the Berlin and the Epworth questionnaires, and also the STOP-BANG questionnaire (SBQ) to determine the risk of suffering from OSAHS. Also analyzed is a validated questionnaire called GAIAS. The latter has been developed by an Argentine team which looked at symptoms and factors that promote the development of this disease.

The usefulness of these questionnaires lies in the interpretation of each of them, being this the object of the presentation.

Keywords: drowsiness, questionnaires, sleep apneas.

\section{INTRODUCCIÓN}

El síndrome de apneas-hipopneas obstructivas durante el sueño (SAHOS) representa un problema de salud pública, tanto por su elevada prevalencia en la población general como por el importante aumento de la morbimortalidad que conlleva ${ }^{1}$.

Si se considera como criterio diagnóstico la presencia de un índice de apneas-hipopneas (IAH) superior a 5 eventos/hora asociado a excesiva somnolencia diurna o a enfermedad cardíaca o metabólica, su prevalencia en

I. Centro de Medicina Respiratoria. Hospital Británico de Buenos Aires. 2. Unidad de Sueño y Ventilación. Hospital Británico de Buenos Aires.

3. Departamento de Docencia e Investigación. Hospital Británico de Buenos Aires.

Correspondencia: Dr. Eduardo Borsini. Servicio de Medicina Respiratoria, Hospital Británico de Buenos Aires, Perdriel 74, CI280AEB CABA, Rep. Argentina. Tel: +54 I I-43096400, Ext: 2808. Email: eborsini@hbritanico.com.ar

Los autores declaran no poseer conflictos de intereses.

Recibido: 21/06/2018 | Aceptado: 13/07/2018
Latinoamérica se estima en alrededor de un $28 \%$ en individuos de mediana edad ${ }^{2}$.

El diagnóstico de SAHOS se confirma convencionalmente mediante una polisomnografía (PSG), aunque se acepta como válido el diagnóstico mediante una poligrafía respiratoria (PR) convenientemente validada en poblaciones con alta o baja probabilidad clínica de padecer la enfermedad ${ }^{3-4}$.

Teniendo en cuenta la documentada relación entre el SAHOS y un incremento en la probabilidad de entre 2 a 7 veces de sufrir un accidente de tránsito, un aumento de la morbimortalidad de origen cardiovascular y la alta efectividad del tratamiento con presión positiva continua de la vía aérea (CPAP), parece prioritario el estudio de variables clínicas de aproximación diagnóstica en la población que asiste a nuestras unidades con el fin de identificar las formas severas y priorizar los recursos diagnósticos disponibles para ese grupo de pacientes ${ }^{5,6}$. Diversos autores han propuesto diferentes opciones en este sentido, como la valoración mediante la utilización de parámetros clínicos, funcionales o antropométricos, con el fin de identificar la probabilidad del paciente de padecer SAHOS de relevancia o para intentar calcular su índice de apneas e hipopneas por hora (IAH) ${ }^{7}$ ${ }^{10}$. Dentro de este espectro de opciones, las estrategias 
más estudiadas han sido sin duda los parámetros clínicos. Varios estudios han valorado su papel como herramienta diagnóstica con la construcción de ecuaciones de predicción mediante métodos multivariados ${ }^{7-12}$. La gran combinación de variables posibles y la heterogeneidad de las poblaciones en las que se han aplicado dificultan las comparaciones o extrapolaciones.

Si bien los resultados han sido dispares, por lo general las ecuaciones de cálculo o predicción han presentado una elevada sensibilidad (entre el 78 y el 95\%) y una escasa especificidad (entre el 41 y el 63\%) para puntos de corte variables en el IAH en diferentes estudios, por lo general entre 5 y 20, y diferente prevalencia de SAHOS en las poblaciones analizadas ${ }^{13-14}$.

Algunas sociedades científicas en la materia alrededor del mundo han establecido un límite arbitrario de 30 eventos/hora en el IAH para distinguir aquellos pacientes que, dependiendo de su sintomatología y antecedentes cardiovasculares, son susceptibles de tratamiento con CPAP. La exigencia de un valor fijo de IAH que permita indicar el tratamiento ha comenzado a cuestionarse en los últimos años, aunque el valor de IAH $\geq 15$ continúa utilizándose para la indicación de terapia con CPAP en las recomendaciones de diferentes sociedades científicas en la materia ${ }^{14}$. La identificación de los pacientes con una alta probabilidad de tener un $\mathrm{IAH} \geq 15$ tendría una aplicación interesante, ya que agrupa a individuos con trastornos moderados y severos, candidatos a ser tratados independientemente de la modalidad terapéutica finalmente elegida según nuestro entendimiento actual sobre la enfermedad, y permitiría priorizar a estos pacientes a la hora de realizar el estudio diagnóstico.

Algunos cuestionarios simples pueden ser utilizados para identificar a los pacientes con alta probabilidad clínica de presentar trastornos respiratorios del sueño. Estos cuestionarios pueden complementarse entre sí en diferentes combinaciones, no siendo mutuamente excluyentes. Su sensibilidad (S) y especificidad (E) en pacientes que concurren a unidades especializadas (Unidades de Sueño o Laboratorios) ha sido descripta en nuestro medio y son rutinariamente aplicados en la Unidad de Sueño del Hospital Británico ${ }^{15-22}$.

\section{CUESTIONARIOS DE BERLÍNY EPWORTH} PARA DETERMINAR RIESGO DE PADECER SAHOS

Por la subjetividad que presentan los síntomas de cansancio y somnolencia, se han validado diferentes test/ cuestionarios que colaboran a objetivar los síntomas y la probabilidad de presentar diagnóstico de SAHOS ${ }^{15-22}$.

El más utilizado es el cuestionario de Berlín, el cual agrupa las preguntas en tres categorías: la primera valora la presencia de ronquidos y apneas nocturnas, la segunda valora el cansancio y la somnolencia y finalmen- te la tercera categoría la presencia de HTA y el valor del índice de masa corporal. Ver anexo en sitio de la revista. Aquellos pacientes que señalan 2 o más categorías presentan alta probabilidad de desarrollar SAHOS. Se ha descripto una sensibilidad del $69.9 \%$ y una especificidad del $56.4 \%$, para un IAH $>5$, aumentando a $72.2 \%$ y $46.4 \%$ respectivamente en aquellos pacientes que presentan un IAH superior a 30 eventos/hora.

La escala de somnolencia subjetiva de Epworth (ESS) diseñada para ser autoadministrada, consta de ocho preguntas que evalúan diferentes situaciones de la vida diaria en las cuales el paciente establece la posibilidad de dormirse realizando las mismas ${ }^{23-24}$. Ver anexo en sitio de la revista.

Ha sido demostrada la utilidad para el diagnóstico de somnolencia excesiva en aquellos pacientes que presentan una elevada puntuación (mayor de 11 sobre 24 puntos posibles). Numerosos estudios han considerado los beneficios y las desventajas de Epworth con grandes diferencias. Sin embargo, la evidencia disponible le otorga valores de eficacia relativamente bajos. Entre sus limitaciones se puede identificar que la excesiva somnolencia diurna es un síntoma subjetivo y difícil de medir. Este test presenta una sensibilidad del $70 \%$ y una especificidad del $56 \%$, para un IAH mayor a 5 eventos/hora, aumentando a $72 \%$ y $46 \%$ respectivamente en aquellos pacientes que presentan un IAH superior a 30 eventos/ hora. En nuestro medio, ESS tuvo una modesta contribución para identificar pacientes con SAHOS ya que solo un tercio de la población presenta más de 11 puntos cuando esta escala es usada de manera sistemática. El área bajo la curva obtenida entre ESS e IAH fue de 0.56 (mínima contribución) para el punto de corte con mejor desempeño en nuestra población (11 puntos) $)^{15}$

Un estudio del Hospital Británico en el 2017, en muestra analizada de pacientes evaluados en un centro de HTA a quienes se indicó una poligrafía respiratoria domiciliaria, presentó predominio de hombres de mediana edad con sobrepeso u obesidad, y una alta prevalencia de trastornos respiratorios del sueño evidenciada por un IAH global elevado (18 eventos/hora) y al hecho de que el $50 \%$ de los sujetos estudiados tuvieron un IAH > 15 eventos/hora en la PR. Solo el 30\% de los sujetos estudiados presentaron ESS $>11$ y no existió correlación entre ESS y los hallazgos cardiológicos (HTA, MAPA) o el IAH en la PR ambulatoria ${ }^{16}$.

A pesar de estas limitaciones, la escala de somnolencia subjetiva de Epworth se recomienda durante la evaluación comprensiva del sueño y puede ser útil para identificar individuos con somnolencia ${ }^{14,25}$. Numerosos pacientes con trastornos respiratorios del sueño no relatan somnolencia clara, aunque relatan síntomas subjetivos inespecíficos o poco característicos referidos como cansancio, falta de energía, escaso rendimiento duran- 
te el día o expresan quejas de sueño poco reparador o de mala calidad. A pesar de que ESS no evalúa completamente estos aspectos, herramientas que permitan admitir la opción de "cansancio" o "fatiga" durante el cuestionario pueden ser interesantes a la hora de identificar pacientes con SAHOS. En nuestro medio hemos hallado que el $58.6 \%$ de los pacientes relatan "cansancio" ( $T$ : tired $)^{16,17,19}$.

\section{CUESTIONARIO DE STOP-BANG (SBQ)}

Otro cuestionario es el denominado por su mnemotecnia (en inglés) STOP-BANG, que consta de 8 preguntas, con una sensibilidad del 93 al 100\% y una especificidad del $43 \%$ para el diagnóstico de SAHOS. Borsini et al., en Argentina, estudiaron la utilidad del cuestionario STOP-BANG con poligrafía respiratoria y demostraron que la mejor relación sensibilidad y especificidad para la identificación de pacientes con SAHOS se obtuvo con tres componentes de STOP en cualquier combinación posible (S: 52.9\%; E: 60.0\%) y con dos componentes de BANG (S: 79.0\%; E: 43.7\%). Para los pacientes con SAHOS severo la mejor relación sensibilidad y especificidad se obtuvo con dos componentes de STOP (S: 79.0\%; E: 43.7\%) y 3 componentes de BANG (S: 61.7\%; E: 65.4\%). Aquellos que presentaron cinco componentes del STOP-BANG (cualquier combinación) alcanzaron una sensibilidad de $60.7 \%$ y especificidad de $65 \%{ }^{17-19}$.

SBQ puede mostrar un desempeño diferente cuando se analizan poblaciones no quirúrgicas, e interesa conocer su valor de discriminación en pacientes hipertensos que son tradicionalmente considerados de alto riesgo. Hemos hallado un valor de AUC-ROC 0.76 en una población derivada por sospecha clínica con 5 componentes en cualquier combinación ${ }^{17,19}$. Este hallazgo permitiría priorizar individuos de alto riesgo a PR preferente. La metodología empleada para el diagnóstico o el tipo de población pueden modificar la predicción del cuestionario, como lo ha propuesto Silva, usando PSG domiciliaria quien comunica que el desempeño de SBQ difieren del trabajo de validación original, posiblemente por tratarse de poblaciones fundamentalmente clínicas ${ }^{26,27}$.

Numerosos cuestionarios han sido propuestos para la búsqueda de pacientes con SAHOS y aún no es claro cuál resulta más eficaz. En un metaanálisis, Ramachandran y Josephs compararon instrumentos multiparamétricos para identificar pacientes con apneas del sueño, incluyendo; cuestionario de Berlín,
ESS, Sleep disorder questionnaire (SDQ), ASA score (American Society of Anesthesiologist checklist) y SBQ, concluyendo que Berlín y SDQ fueron más eficaces para identificar $\mathrm{SAHOS}^{28}$. La revisión sistemática de la literatura realizada por Abrishami et al. comunica conclusiones similares. Sin embargo, reconoce que es posible que el uso concomitante de más de una herramienta de predicción mejore los resultados y que SBQ aporta datos de alta calidad con resultados de eficacia razonable ${ }^{29}$.

En este sentido, los autores del trabajo basado en participantes del Sleep Heart Health Study sostienen que SBQ puede representar una herramienta útil para descartar SAHOS (umbral de IAH $<5$ eventos/hora) en poblaciones de bajo riesgo (población general).

Finalmente, investigadores argentinos han validado un cuestionario específico (Cuestionario GAIAS) en pacientes que asisten a dos Unidades de Sueño metropolitanas para la realización de una prueba de sueño, demostrando que es posible indicar empíricamente terapia con CPAP a un tercio de la población de riesgo con alta especificidad (>98\%). Ver anexo en sitio de la revista.

Es de resaltar que es posible realizar de manera sistemática una combinación de cuestionarios (Berlín + ESS + STOP-BANG) en pacientes derivados para evaluación por sospecha de alteraciones respiratorias del sueño. Estos cuestionarios incluidos en un formulario específico (ver anexo) se usa rutinariamente previo a la entrega de PR y PSG junto con un cuestionario comprensivo de sueño antes de la PSG. Se propone el cuestionario GAIAS para fines de investigación. Ver anexo en sitio de la revista.

\section{NOTAS}

El cuestionario de ESS debe ser escrito y autoadministrado. Pacientes que no tengan un desempeño motor o visual o que no conduzcan vehículos no son candidatos a completarlo ya que su interpretación no será válida en esta situación.

Los cuestionarios de Berlín y STOP-BANG podrán ser completados entre personal médico o técnico entrenado y el/la paciente. La interpretación será realizada por el personal médico o técnico a cargo.

El cuestionario de Berlín se interpretará dicotómicamente como ALTO o BAJO riesgo de padecer apneas del sueño.

Se deberán consignar los componentes positivos de STOP-BANG en el reporte de los estudios de sueño. 


\section{BIBLIOGRAFÍA}

I. Phillipson DE. Sleep apnea.A major public health problem. N Engl J Med 1993; 328:127|-3.

2. TufikS, Santos-Silva R,Taddei JA, Bittencourt LR. Obstructive sleep apnea syndrome in the Sao Paulo Epidemiologic Sleep Study. Sleep Med. $2010 ;$ I I (5):44I-6.

3. Grupo Español de Sueño. Documento consenso español sobre el síndrome de apneas-hipopneas del sueño. Arch Bronc 2005; 4 I (S4):3-1 10

4. Clinical Guidelines for the Use of Unattended Portable Monitors in the Diagnosis of Obstructive Sleep Apnea in Adult Patients. Portable Monitoring Task Force of the American Academy of Sleep Medicine. Journal of Clinical Sleep Medicine 2007;3(7):737-47.

5. Teran-Santos J, Jimenez-Gomez A, Cordero-Guevara J.The association between sleep apnea and the risk of traffic accidents. $\mathrm{N}$ Engl J Med 1999;340:847-51.

6. Leung RST, Bradley TD. Sleep apnea and cardiovascular disease. Am J Respir Crit Care Med 200 I; 164:2147-65.

7. Deegan PC, McNicholas WT. Predictive value of clinical features for the obstructive sleep apnoea syndrome. Eur Respir J 1998;9: | 17-24

8. Rowley JA, Aboussouan LS, Badr MS. The use of clinical prediction formulas in the evaluation of obstructive sleep apnea. Sleep 2000;23:929-37.

9. Zerah-Lancner F, Lofaso F, D'Ortho MP, Delclaux C, Goldenberg $F$, Coste A, et al. Predictive value of pulmonary function parameters for sleep apnea syndrome. Am J Respir Crit Care Med 2000; 1 62:2208-12.

10. Kushida CA, Efron B, Guilleminault C. A predictive morphometric model for the obstructive sleep apnea syndrome. Ann Intern Med 1997; 127:581-7.

I I. Hofftein V, Szalai JP. Predictive value of clinical features in diagnosing obstructive sleep apnea. Sleep 1993; 16: I | 8-22.

12. Rowley JA, Aboussouan LS, Badr MS. The use of clinical prediction formulas in the evaluation of obstructive sleep apnea. Sleep 2000;23:929-37.

13. Flemmons WW. Obstructive Sleep apnea. N Engl J Med 2002; 347: 498-501.

14. Sala H, Nigro C, Rabec C, Guardia AS, Smurra M. Consenso argentino de trastornos respiratorios vinculados al sueño. Medicina (B Aires) 2001;61:351-63.

15. Borsini E, Delgado Viteri C Chertcoff M, et al. Relación entre la escala de somnolencia de Epworth y el índice de apneas e hipopneas de la poligrafía respiratoria. Borsini et al. RAMR 2013;3; I 23- I 32.
16. Borsini E, Blanco M, Bosio M, Schrappe M, et al. Prevalence of sleep apnea and cardiovascular risk factors in patients with hypertension in a day hospital model. Clin Exp Hypertens 2017;5: I-7.

17. Borsini E, Ernst G, Salvado A, et al.Utility of the STOP-BANG components to identify sleep apnea using home respiratory polygraphy. Sleep Breath 2015;19(4): I 327-33.

18. Borsini E, Maldonado L, Decima T, et al. Predictores clínicos de $|A H \geq|$ 5/hora en la poligrafía respiratoria. RAMR 20 | 2;4:90-7.

19. Borsini E, Salvado A, Bosio M, et al. Utilidad de los componentes del cuestionario Stop-Bang para identificar pacientes con apneas del sueño. RAMR 20 14:4:382-403.

20. Ernst G, Salvado A, Bosio M, et al.Relación entre el índice de masa corporal, sexo, edad y anormalidades respiratorias durante el sueño. RAMR 2015;3:203-212.

21. Borsini E, Ernst G, Bosio M, Blanco M, Blasco M, Salvado A. Role of sex and age as predictors of an apnea and hypopnea index higher than 5/hour in patients with normal body mass index using respiratory polygraphy. MedClin (Barc)2016; 7; 147(7):293-6.

22. Nigro CA, Dibur E, Borsini $E$, et al. The influence of gender on symptoms associated with obstructive sleep apnea. Sleep Breath. 20 I8. En prensa (Doi: 10.1007/s | |325-017-1612-4).

23. Johns MW. Daytime sleepiness, snoring and obstructive sleep apnea. The Epworth Sleepiness Scale. Chest 1993; I03(I):30-6.

24. Izquierdo-VicarioY, Ramos-Platon MJ, Conesa-Peraleja D, LozanoParra AB, Espinar-Sierra J. Epworth Sleepiness Scale in a sample of the Spanish population. Sleep 1997;20(8):676-7.

25. Nogueira F, Nigro C, Cambursano H, Borsini E, Silio J, Avila J. Practical guidelines for the diagnosis and treatment of obstructive sleep apnea syndrome. Medicina (B Aires) 2013;73(4):349-62.

26. Silva GE, Vana KD, Goldberg R. Predictive abilities of the STOPBang and Epworth Sleepiness Scale in identifying sleep clinic patients at risk for obstructive sleep apnea. Res Nurs Health 2013;36(I):84-94

27. Chung F,Yegneswaran B, Liao P, et al.Validation of the Berlin questionnaire and American Society of Anesthesiologists checklist as screening tools for obstructive sleep apnea in surgical patients. Anesthesiology 2008; 108(5):822-30.

28. Ramachandran SK, Josephs $L A$.A meta-analysis of clinical screening tests for obstructive sleep apnea. Anesthesiology 2009; I 10:92839.

29. Abrishami A, Khajehdehi A, Chung F.A systematic review of screening questionnaires for obstructive sleep apnea. Can J Anaesth 2010;57:423-38 